\title{
Giant Thoracocervical Lymphangioma with Multivisceral Involvement: A Different Concept
}

\author{
${ }_{1}^{1}$ G Raghavendra Prasad, ${ }^{2}$ Madhavi Nori, ${ }^{3}$ MD Naseeruddin, ${ }^{4} \mathrm{JV}$ Subba Rao, ${ }^{5}$ SS Quadri
}

\begin{abstract}
Background: Lymphangiomas are benign lymphatic malformations frequently seen in head and neck region. Most reported cases are cervical lesions with mediastinal extension..$^{1,2}$

Most are conceived as due to failure of fetal lymph sacs. Synchronous or metachronus lymphangiomatosis with cystic hygroma of the neck is also reported. ${ }^{3}$
\end{abstract}

Case details: Massive multicompartmental mediastinal lymphangioma with cervical extension and concomitant visceral involvement is presented. It is proposed as a hamartomatous tumor rather than malunion of fetal jugular lymphatic sacs. ${ }^{2}$ Solid intrathoracic component with cystic neck extension supports mediastinal origin. Cell culture lines at 4 months were positive for endothelial cell lines positive for factor VIII antigen.

Conclusion: Giant thoracocervical lymphangioma is more likely to be a tumor rather than simple fetal failure of lymphatic sac fusion. Surgical excision is curative.

Keywords: Giant thoracocervical lymphangioma, Multivisceral involvement, Magnetic resonance imaging.

How to cite this article: Prasad GR, Nori M, Naseeruddin MD Rao JVS, Quadri SS. Giant Thoracocervical Lymphangioma with Multivisceral Involvement: A Different Concept. Int J Phonosurg Laryngol 2014;4(2):63-66.

Source of support: Nil

Conflict of interest: None

\section{INTRODUCTION}

Lymphangiomas are benign lymphatic malformations frequently seen in head and neck region. Most reported cases are cervical lesions with mediastinal extension. ${ }^{1,2}$ Synchronous or metachronus lymphangiomatosis with cystic hygroma of the neck is also reported. ${ }^{3}$ Massive multicompartmental mediastinal lymphangioma with cervical

\footnotetext{
${ }^{1}$ Professor, ${ }^{2,4,5}$ Associate Professor, ${ }^{3}$ Senior Resident

${ }^{1}$ Department of Pediatric Surgery, Deccan College of Medical Sciences, Hyderabad, Telangana, India

${ }^{2,3}$ Department of Radiology, Deccan College of Medical Sciences, Hyderabad, Telangana, India

${ }^{4}$ Department of Anesthesia, Deccan College of Medical Sciences, Hyderabad, Telangana, India

${ }^{5}$ Department of Pathology, Deccan College of Medical Sciences, Hyderabad, Telangana, India
}

Corresponding Author: G Raghavendra Prasad, Professor Department of Pediatric Surgery, Deccan College of Medical Sciences, Hyderabad, Telangana, India, Phone: 919849096145 e-mail: grprasad22@gmail.com extension and concomitant visceral involvement is presented. It is proposed as a hamartomatous tumor rather than maldevelopment of fetal jugular lymphatic sacs. ${ }^{2}$ Solid intrathoracic component with cystic neck extension supports mediastinal origin. Magnetic resonance imaging (MRI) and other radiological findings were correlated with clinical, surgical and histological features.

\section{CASE REPORT}

A 17-year-old boy presented with painless swelling in left side of the neck associated with heaviness and discomfort in the chest of 4 years' duration. There was no history of dyspnea, dysphagia or repeated chest infections.

The swelling in the neck was supraclavicular and deep to sternocleidomastoid. It was soft, cystic, fluctuant and transilluminant. Lower pole of mass could not be reached (Fig. 1A).

Chest radiograph lateral view (Fig. 1B) revealed large homogeneous opacity occupying anterior and middle mediastinum with compression of left lung posteriorly. Ultrasound scan of neck showed a $4.5 \times 1.5 \mathrm{~cm}$ cystic lesion with regular margins, multiple internal septae, avascular signal on Colour Doppler extending intrathoracically (Fig. 1C). The mass was not compressing the barium filled esophagus (Fig. 1D). Magnetic resonance imaging revealed a thoracocervical mass of $25 \times 14 \times 11 \mathrm{~cm}$ size. It was occupying the anterosuperior, anterior and middle mediastinal compartments compressing the lung posteriorly. It extended from the left hemidiaphragm to lower neck without displacement of major mediastinal vessels (Fig. 2A).

The lesion extended to left half of the neck through the thoracic outlet. Superficial subplatysmal component and deep substernomastoid posterior triangle component formed a dumbbell. Internal jugular vein was surrounded by the mass (Fig. 2B). The mass was multilocular, septate with smooth contours and thick walls. It was isointense (to skeletal muscle) on T1-weighted images with intermittent hyperintense signals signifying solid cystic nature; T2-weighted images showed hyperintensity. Subplatysmal component was homogenous as against intrathoracic component. Mass was separated from the surrounding structures without compression. 

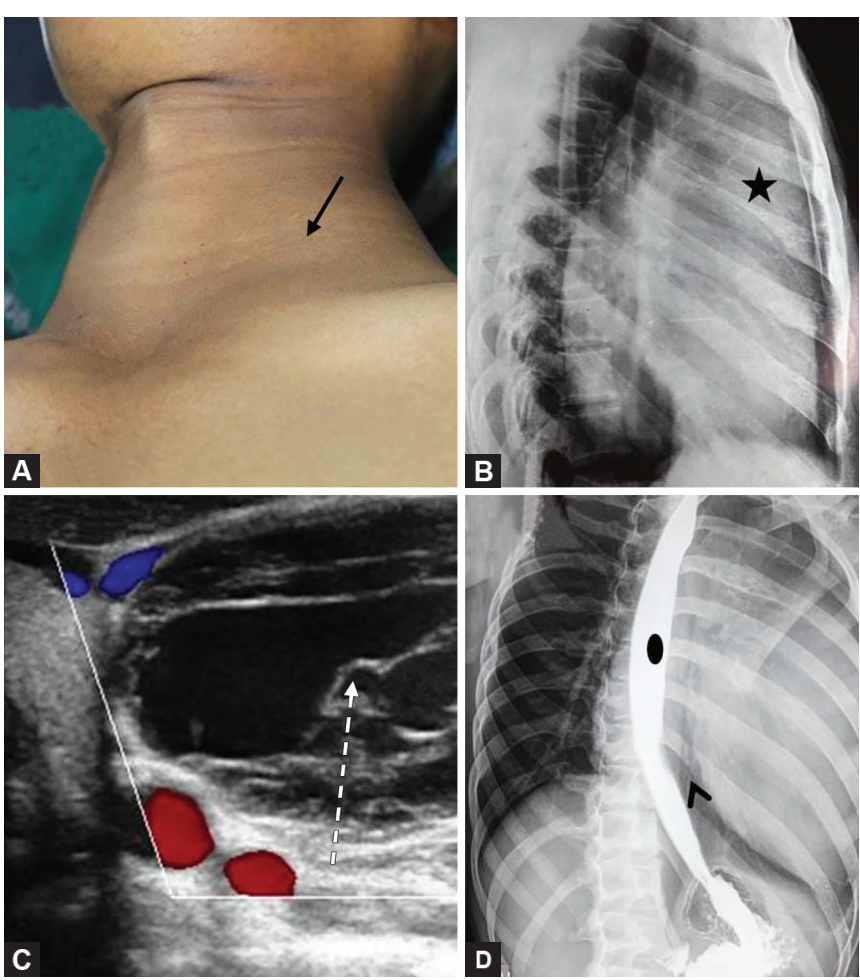

Figs 1A to D: (A) Soft cystic fluctuant transilluminant mass present in lower part of neck in posterior triangle (arrow). Chest radiograph lateral view (B) shows homogeneous opacity occupying anterior and middle mediastinum (star). USG with Color Doppler (C) 4.5 $\mathrm{cm} \times 1.5 \mathrm{~cm}$ capsulated anechoic lesion with regular margins and multiple internal septa (dashed arrow), with no vascular signal, at the level of the left lower neck. Barium swallow AP (D) revealed no mass effect on esophagus (black dot). Left lung compressed inferiorly (arrowhead)

Further MRI also showed T2 hyperintense lesions in the spleen, vertebral bodies, ribs, the upper and lower extremity bones and bones of pelvis (Fig. 2C) without bone marrow edema, periosteal reaction or accompanying soft-tissue mass. Computed tomography (CT) scan of the chest (Fig. 2D) revealed low density lesion with attenuation values ranging between 18 and $36 \mathrm{HU}$ with multiple internal septae. No calcification was seen within the lesion.

Surgical exploration revealed superficial subplatysmal component and deep substernomastoid posterior triangle component having a dumbbell connection. Retrosternocleidomastoid swelling was compressing the IJV medially. Extension into mediastinum behind manubrium sterni did not show pressure effect on trachea and esophagus. Mass was large and solid occupying entire left hemi-thorax necessitating additional left anterior thoracotomy as solid nature prevented decompression. Mass was excised sparing the phrenic nerve (Fig. 2E).

Recovery was uneventful and left lung expanded completely. Histopathology was hamartoma with neural component. Cultured cells from mass at 4 months showed a viable primary endothelial cell line positive for factor VIII-associated antigen.

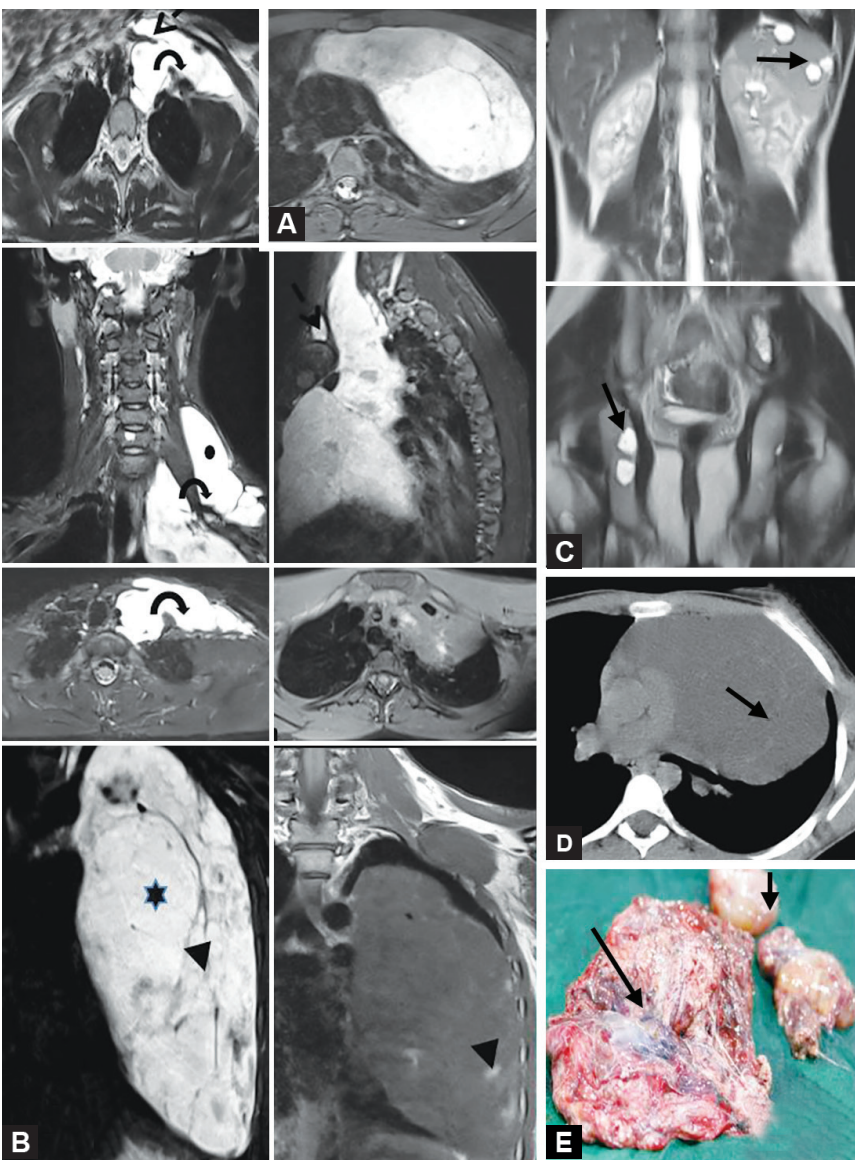

Figs 2A to E: Axial, coronal and sagittal T2W MRI images (A): Mass showing cervicothoracic involvement. Left lung compressed posteroinferiorly. The lesion envelops the ascending aorta and main pulmonary artery without compressing them; Low signal intensity of the vessels is well-differentiated by the mass. (B) T2W axial, coronal, and sagittal MRI: $25 \mathrm{~cm} \times 14 \mathrm{~cm}$ $\times 11 \mathrm{~cm}$ mass shows isointense (to skeletal muscle) signal on T1-weighted images with intermittent hyperintense signals (bold arrow) and hyperintense signal on T2-weighted images (arrow) with heterogeneous intrathoracic component (star). Dumbbell connection of superficial subplatysmal component (dashed arrow) and deep substernomastoid posterior triangle component (black dot) to axilla (curved arrow). (C) Coronal HASTE MRI: Additional T2 hyperintense lesions in the spleen (arrowhead), vertebral bodies, ribs, the upper and lower extremity bones and bones of pelvis (arrow) without bone marrow edema, periosteal reaction and accompanying soft tissue mass. (D) CT scan reveals multiseptate low density mediastinal mass without calcification (short arrow) (E) Operative specimen shows heterogeneous intrathoracic component (long arrow) with cystic cervical component (arrowhead)

\section{DISCUSSION}

Cystic lymphangiomas, first described by Rodenber in $1828,{ }^{3}$ are rare benign lymphatic tumors with equal distribution among genders and races. ${ }^{1,3}$ Ninety percent of lymphangiomas develop on body surface, $75 \%$ in neck, mostly posterior triangle and $20 \%$ in axilla. Most are detected before 2 years of age. ${ }^{1,2}$ One percent of cystic lymphangiomas have mediastinal localization. ${ }^{4}$ Frequent locations are the anterior and upper mediastinum $(50-60 \%)$ with rare occurrence in the middle and posterior 
mediastinum. ${ }^{4}$ Cystic lymphangioma of the mediastinum in the right paratracheal, paracardiac, anterior and posterior mediastinal regions have been reported. ${ }^{5-7}$ Less frequent sites are in the adrenal gland, kidney, bone, omentum, gastrointestinal tract, retroperitoneum, spleen, liver and pancreas. ${ }^{6}$ They grow proportional to patient's body growth. ${ }^{3}$ There are only few reports of giant mediastinal lymphangiomas. ${ }^{5,7}$ Whether lymphangiomas are true malformations or tumors is not addressed comprehensively in literature. $^{8}$

Lymphangiomas are conventionally hypothesized to be hamartomas representing sequestered lymphatic sacs which fail to communicate with the remainder of the lymphatic or venous system, during the development in 8th week of gestation. Subsequently, sequestered lymphatic tissues lead to cystic morphology. ${ }^{1-3,9}$

The expression of different growth factors in the endothelium of lymphangiomas supports tumoral origin. Bowman et al hypothesized that cystic hygroma represents an expanding proliferating endothelial growth process and not simply a sequestered lymphatic receptacle. $^{8}$

Lymphangiomas traditionally are divided into capillary, cavernous or cystic, ${ }^{1}$ and macrocystic, micro-cystic or combined, on gross morphology. ${ }^{8}$

Histopathology showed lymphangioma with single layer of flattened endothelium lined spaces filled with eosinophilic proteinaceous material between fat, fibrotic and neural and lymphatic structures. ${ }^{3,9}$ Immunochemistry confirms the endothelial line of differentiation in lymphangiomas. Cells cultured from the mass yielded an endothelial cell line positive for factor VIII-associated antigen. $^{8}$

Solid mediastinal component with skeletal muscle intensity on MR and inability to decompress at surgery indicate tumor behavior in the present case.

Site of primary focus (chest or neck) is a controversy ignored in previous publications. All cervicothoracic lesions except superficial lesions extend from the neck to the thorax through the thoracic inlet. ${ }^{1}$ In the present case, we strongly believe in an intrathoracic origin because of greater intrathoracic component (>95\%). Only the cystic component extended along neurovascular planes into the neck. If it was the other way round, chest component also would have been cystic. Normally, when lymphangiomas extend, they go behind the lung. Solid intrathoracic component was anterior, pushing the lung posteriorly. Imaging findings also support below upwards migration; more of chest component extending into neck due to lack of space.

The issue of disseminated lymphangiomatosis vs diffuse lymphangiomatosis with associated giant lymphangioma also deserves mention. The former represents a proliferation of lymph vessels involving soft tissue skin and the skeletal system. Clinical presentation depends on the organ involved and extent of the mass. Diffuse lymphangiomatosis occurring synchronously or metachronously with cystic hygroma of the neck is occasionally reported. ${ }^{3}$

Giant intrathoracic solid component and cystic cervical part with patchy multivisceral involvement distinguishes the present case from disseminated lymphangiomatosis.

Integrating the ultrasound (US), CT and MRI scan findings yields comprehensive anatomical details. ${ }^{3}$ The common feature in the diagnosis of lymphangiomas are uni- or multilocular predominantly cystic masses, clear borders, septations with lobular contours, contiguous extension along neurovascular planes of mediastinum without mass effect. Magnetic resonance imaging in cystic hygromas is fundamental and indispensable because of its advantages. ${ }^{3,10}$ Multiplanar, nonionising, high contrast resolution with tissue-specific signal intensity provides accurate anatomy and internal structure of the lesion.

Lymphangiomas are hyperintense to fat on T2-weighted MR images, and are isointense to muscle on T1-weighted. High signal intensity on T1 represents elevated protein content in the fluid or combination of fluid, solid tissue and fat. ${ }^{3}$

In the present case, MRI showed a cystic-solid septate lesion with detailed anatomy, in particular, the close proximity to the brachiocephalic and common carotid arteries. A large predominantly solid intrathoracic lymphangioma with cystic extension into neck presented as a complex mass. Hence, a tumor origin with solid components is preferred to conventional fetal nonunion of lymph sacs.

The treatment options of intrathoracic lymphangiomas also range from surgical excision to intralesional sclerosant to radiotherapy., ${ }^{9,11}$ Surgical treatment is the gold standard and preferred by many. ${ }^{10,12}$

The bigger the lesion, the greater is the technical difficulty of excision. Surgeon's mindset regarding challenging dissection around the vital structures supported the use of intralesional sclerosant agents. ${ }^{11}$ Surgical excision was preferred in the present case due to solid cystic nature of the mass.

Visceral involvement (splenic/bony) are kept under observation for growth and symptoms expecting spontaneous resolution.

\section{CONCLUSION}

Giant solid cystic intrathoracic lymphangioma with extension into neck with multivisceral involvement is a rare complex presentation. Tri-dimensional MRI provides accurate anatomy, especially of local and distal involvement. Complex solid nature supports tumor rather than 
sequestered fetal lymphatic channels and lead to option of surgery.

Immunochemistry confirms the endothelial line of differentiation in lymphangiomas. Tissue cultures of explants yield a primary endothelial cell line positive factor VIII-associated antigen.

\section{ACKNOWLEDGMENT}

The authors thank the administration of Deccan College of Medical Sciences for permitting access to the case sheet and publication of the report.

\section{REFERENCES}

1. Ravitch MM, Rush BF Jr. Cystic hygroma. In: Paediatric surgery. Year Book Medical Publishers Inc, Chicago 1986;1:536.

2. Grasso DL, Pelizzo G, Zocconi E, Schleef J. Lymphangiomas of the head and neck in children. Acta Otorhinolaryngol Ital 2008;28(1):17-20.

3. Ozel A, Uysal E, Dokucu AI, Erturk SM, Basak M, Cantisani V. US, CT and MRI findings in a case of diffuse lymphangiomatosis and cystic hygroma. J Ultrasound 2008 Mar;11(1):22-25.

4. Teramoto K, Suzumura Y. Mediastinal cavernous lymphangioma in an adult. Gen Thorac Cardiovasc Surg 2008 Feb;56(2):88-90.
5. Bossert T, Gummert JF, Mohr FW. Giant cystic lymphangioma of the mediastinum. Eur J Cardiothorac Surg 2002;21(2):340.

6. Yildirim E, Dural K, Kaplan T, et al. Cystic lymphangioma: report of two atypical cases. Interact Cardiovasc Thorac Surg 2004;3(1):63-65.

7. El Murr T, Youssef P, Khairallah S, Sleilaty F, Ghayad E. Giant mediastinal cystic lymphangioma: clinical case and review of the literature. J Med Liban 2006 Mar;54(1):54-56.

8. Bowman CA, Witte MH, Witte CL, et al. Cystichygroma reconsidered: hamartoma or neoplasm? Primary culture of an endothelial cell line from a massive cervico mediastinal hygroma with bony lymphangiomatosis. Lymphology 1984 Mar;17(1):15-22.

9. Kennedy TL. Cystic hygroma-lymphangioma: a rare and still unclear entity. Laryngoscope 1989 Oct;99(10 Pt 2 Suppl 49):1-10.

10. Sermon A, Gruwez JA, Lateur L, De Wever I. The importance of magnetic resonance imaging in the diagnosis and treatment of diffuse lymphangioma. Acta Chir Belg 1999 Oct; 99(5):230-235.

11. Colbert SD, Seager L, Haider F, Evans BT, Anand R, Brennan PA. Lymphatic malformations of the head and neck-current concepts in management. Br J Oral Maxillofac Surg 2013 Mar; 51(2):98-102.

12. Rakotosamimanana J, RaharisoloVololonantenaina CR, Ratovoson H, Ahmad A, Razafindramboa H. Cystic cervical and mediastinal lymphangioma: a case report and review of the literature. Arch Inst Pasteur Madagascar 2000; 66(1-2):61-64. 